\title{
La palabra acto tejedora de comunidad ${ }^{3}$
}

\author{
The word act, a weaver of community
}

\begin{abstract}
Resumen
El ejercicio reflexivo que presenta el escrito es producto de inquietudes teóricas sobre la relación entre pedagogía y comunidad, y en especial, sobre el lugar que esta última ocupa en los procesos comunitarios, en los cuales la palabra juega un papel importante en la construcción de propuestas alternativas dialogantes con la diferencia y acompañantes de la democracia. La reflexión se centra en mirar el lugar de la pedagogía en el contexto comunitario desde el cuidar la palabra acto, que pasa por el compromiso de saber ser experiencia de sí, donde el ser amigo de esa palabra hace emerger subjetividades comprometidas con comunidades edificadoras; y desde lo singular de los acontecimientos de las condiciones humanas de vida, de compromisos éticos y de la política de cara a la vida.

Es una palabra acto que, asumida desde la sinceridad y aparecida en el dispositivo pedagógico del conversatorio grupal, pone a prueba la coherencia de lo que se dice y lo que se hace en el reto de hacer de la comunidad el escenario para exponer la pluralidad de opiniones y para hacer de ella la fuerza constitutiva de saberes agenciadores de gobernabilidades autónomas. Es hacer de lo educativo el riesgo de vivir la experiencia de vida, donde lo pedagógico es la "bisagra" del encuentro entre la ética y la política hacia mundos posibles.
\end{abstract}

Palabras clave: palabra acto, conversatorio, pedagogía, comunidad, mundos posibles.

\begin{abstract}
The thoughtful exercise that is shown in this document displays the results from theoretical concerns about the relationship between education and community, and especially, the place that the last one occupies in Community Processes, where the word plays an important role in the construction of alternative dialoguing proposals, along unlikeness and democracy companions. The reflection is focused on looking at pedagogy in the community context, as of caring the word act, which passes through the commitment of knowing how to be an experience of itself, where being a friend of the word act brings up subjectivities devoted with edifying communities, taking into account the singularity of the events of the buman living conditions, ethical and political responsibilities in the face of life.

It is a word act, taken from sincerity, and looked into the pedagogic device among a discussion group, that tests the consistency of what is said, built in the challenge of making the community the stage to expose the plurality of opinions, and also to make it the constitutive force of governability of autonomous knowledge. It is about to make whatever is educative the risk of living the life experience, where pedagogy is the "binge" of the link between ethics and politics, towards possible worlds.
\end{abstract}

Keywords: The word act, discussion group, education, community, possible worlds.

\section{Recibido: 20 de octubre de 2015, evaluado: 24 de octubre de 2015, aprobado: 17 de noviembre de 2015}

1 CTrabajadora social de la Universidad del Valle. Magíster en Estudios Políticos de la Pontificia Universidad Javeriana. Docente de Universidad Santiago de Cali y de la Corporación Universitaria Minuto de Dios-UNIMINUTO. Correo electrónico: maria.gil00@usc.edu.co.

2 Trabajadora Social de la Universidad del Valle. Magíster en Educación Superior de la Universidad Santiago de Cali. Especialista en investigación social de la Universidad del Valle. Especialista en educación superior. Docente de la Universidad Santiago de Cali. Correo electrónico: marielasanchez@usc.edu.co.

3 Artículo de reflexión sobre el papel de la pedagogía en la comunidad, derivado de procesos de investigación de las autoras. 


\section{Introducción}

El presente artículo busca ubicar trazos conceptuales sobre el lugar de la pedagogía en la comunidad, desarrollando dos aspectos fundamentales para el esclarecimiento de dicho lugar.

1. La comprensión del concepto de comunidad en relación con la biopolítica. Para ello se traen las reflexiones de Roberto Espósito, en especial su mirada de la comunidad como algo que, al exponerse al exterior, desarrolla procesos de inmunidad frente a las amenazas venidas de ese exterior; es como si, al tiempo de mostrarse, de arriesgarse a ser capturada por lo externo, se resistiera a dichas capturas, convirtiendo a la comunidad en la situación numerosa y singular de la existencia humana (Espósito, 2009, p. 57). Desde dicha idea, se pretende dejar planteada la comunidad como procesos de subjetivaciones, que agenciados desde las experiencias de vida, se asoman a un exterior para visibilizar sus saberes y sus propuestas, y, bajo el riesgo que ello implica - desaparecer como propuestas-, se resisten a procesos de dominios para retornar fortalecidos $\mathrm{y}$ transformados en el tejer de mundos posibles.

2. Dichos procesos de exterioridad comunitaria provocan el pensarse el lugar de la pedagogía como experiencia de formación, comprendida no con procesos de ensimismamientos psicológicos ni con experimentaciones, ni mucho menos con la acumulación de verdades objetivas, sujetas todas ellas a la transmisión de conocimientos; sino con el cuidar de la palabra acto, que desde la ética acontece en lo comunitario como viaje que se vive y se lucha en lo posible, siendo ese posible el cuidar dicha palabra para devenir en muchos y dejar de ser lo que se era: sujeto, identidad.

El artículo se desarrolla en tres partes: 1), pensarse la comunidad desde las experiencias que suceden en lo singular de las condiciones humanas de vida; 2), el conversatorio como el dispositivo pedagógico donde la palabra acto aparece; y 3), las conclusiones que se derivan de la reflexión de los dos apartados anteriores.

\section{La comunidad como posibles}

Los discursos sociopolíticos de la actualidad, instalados en la comunidad contemporánea, la han hecho circular como aquel lugar "seguro" y "neutro" al que hay que regresar; como el origen buscado; como el final o el destino a aspirar; como aquel espacio de acuerdos de voluntades; como la unificación de necesidades e intereses de individuos reunidos para tal fin; o, en el mejor de los casos, como la unificación de necesidades e intereses sociales por sujetos de derechos, razonablemente educados para pactar acuerdos salvadores de la ley y el orden social.

La comunidad no es algo a lo que haya que regresar, como quería Rousseau, o a lo que debe de aspirar, como desea Kant, ni tampoco algo que destruir o destruible, como pensaba Hobbes. No es un origen, ni un telos, ni un principio, ni un final, ni un presupuesto ni un destino, sino la condición, a la vez singular y plural, de nuestra existencia finita (Espósito, 2003, p. 58).

Discursos que han dejado de lado la desnudez de la vida, y en este aspecto la reflexión que adelanta Roberto Espósito sobre la comunidad o communitas, suscitan el salto de comprenderla más allá de las seguridades dadas por los sujetosindividuos reunidos para dar soluciones, o responder a las diferentes situaciones y problemas vividos desde propuestas de saberes instituidos por una normatividad, previamente consensuada por las leyes.

Este salto tiene dos provocaciones. De un lado, pone a la comunidad en relación con uno de los debates contemporáneos: la biopolítica. Foucault la concibe en términos de control sobre las poblaciones o sobre los cuerpos de los individuos, donde las racionalidades gubernamentales diseñan las formas y maneras de materializar y concretar dichos controles a través de los dispositivos discursivos, y las singularidades o diferencias sociales se convierten en uno de sus objetivos. Y de otro lado, provoca el ir más allá del establecimiento de controles sociales y ubicar la comunidad en el contexto de la autoconservación de la vida y de las organizaciones comunitarias, donde lo posible es el proceso de transformaciones en cadenas, su sentido mismo (Espósito, 2009, p. 112). 
Cuando se entra en el contexto de la vida, la comunidad emerge no desde la causalidad de las problemáticas, ni como un ente que está en relación mesiánica o de rechazo con la sociedad. Se debe comprenderla como el encuentro de las experiencias y aquí es necesario detenerse un momento sobre la comprensión de la experiencia. En lo que nos acontece - y esto ya lo había señalado Jorge Larrosa-, esta última es aquello que atraviesa la existencia para provocar rupturas y abismos, y nos obliga a exponernos a aquello donde no existen seguridades, sino quizás actitudes y corajes para hacerle frente desde lo que somos, con el fin de dejar de ser lo que se era o han hecho de nosotros. En el trabajo con las comunidades se asiste al encuentro de la multiplicidad de afectaciones contadas, recitadas o dichas desde las experiencias, en donde el sujeto se expone al otro - a lo otro- para deconstruirse como sujeto de seguridades e identidad, y se entra al proceso de exponerse al exterior de lo posible, mostrando la fragilidad de la condición humana.

En otras palabras, la comunidad es aquella condición de la existencia humana que al construir propuestas, las realiza de cara a los otros: esos otros que al exponer sus experiencias de vida crean un exterior donde se muestran o se exponen a decir aquello que no se tolera; y al hacerlo desde el ejercicio del coraje de saber decir lo que se siente se dispone a afectar y ser afectado, para así provocar que aquel otro - no como igual, ni como idéntico, ni como origen, sino como diverso- dé paso a aquel tercero como posible, como diferencia y como pluralidad. Es en este momento, cuando la comunidad se despoja de propiedades y seguridades, y expone sus miradas, sus propuestas y sus saberes, aún a riesgo de desaparecer al momento de decir aquello que no se soporta, lucha y se resiste a aquello instituido por poderes duros; y agencia con las fuerzas afectivas (aquello propio de la comunidad) la creación de propuestas alternativas hacia la potencia de existir.

Es claro que los actos creativos comunitarios suceden en el viaje de las experiencias que pasan por inmunizarse frente a los poderes duros, con lo cual dejan que aparezca lo diferente. En esa medida, la inmunidad forma parte de la comunidad, o mejor, de la sabiduría propia del vivir en comunidad. Por ello la aventura de lo posible - lo exterior- está acompañada por el coraje ético de enfrentar las decisiones del diario vivir, que al sucederse en el borde de la vida y con la cercanía de la muerte, teje decisiones atentas a los absurdos de la condición humana y recobra la sensatez de los actos vitales de la vida en comunidad.

Dicho de otro modo, vivir en comunidad es exponerse y tomar el riesgo de ser afectado por el otro, o los otros. Es disponerse a entablar relaciones afectivas dadas en por el hecho de compartir experiencias sobre las formas y maneras como se viven y se asumen las condiciones de vidas, con la condición de saber arriesgarse a comprender, a dejarse "pinchar" por la afección de aquellos poderes duros, para dejar emerger no ya como sujeto, sino como subjetividades. Estas hacen de lo posible aquellas relaciones que pueden llegar a hacer mundos para vivir dignamente, donde se busquen y se construyan actos creativos en la convivencia con los muchos, con lo diverso. Es decir, es salir de aquellas miradas, de aquellos discursos, evocadores de sujetos esencialistas y totalidades organizacionales para entrar en lo plural, y en las singularidades de los proceso comunitarios.

Esta teoría de las relaciones exteriores, "flotantes", "variadas", "fluidas" nos hace salir del universo de la totalidad y entrar en el mundo del pluralismo y de la singularidad, donde las conjunciones y las disyunciones entre las cosas son en cada momento contingentes, específicas y particulares y no remiten a ninguna esencia, sustancia, estructura profunda que las pudiesen fundar (Lazzarato, 2006, p. 19).

Así mismo, las singularidades remiten a la diferencia del acontecimiento de la existencia de sí, que al poner en situación a las individualidades provoca el compromiso de asumir el presente para dejar de ser lo que se es y crear otras formas de vivir comprometidas con una comunidad para la vida. Estos dos aspectos, o mejor, procesos de la comunidad -lo exterior y el acontecimiento-, hacen de la experiencia un devenir de otro como tercero posible, en el cual en vez de poseer se da y se en- 
frenta el acto de afectar y ser afectado, para asomarse aquel exterior en el que (como experiencias compartidas) se recobra el coraje que da la fuerza afectiva de haber sabido protegerse frente a los miedos, a las amenazas, a las banalidades, al terror instalado en lo cotidiano, al sujeto de propiedad, al sujeto de identidad y al sujeto de derecho, establecidos en discursos cuyo centro es el control de la potencia de existir.

Si la communitas determina la ruptura de las barreras protectoras de la identidad individual, la immunitas es el intento de reconstruirla en una forma defensiva y ofensiva contra todo elemento externo que venga a amenazarla (Espósito, 2003, p. 17).

Es en estos términos donde la inmunidad es un concepto fundamental para la comprensión de la relación entre comunidad y exterior, desde las experiencias que son acontecimientos vividos por los individuos dispuestos a vivir el viaje de construir el orden establecido para provocar otros singulares.

Ahora bien, si la comunidad es exponerse a la exterioridad donde al hacerse resistente a las amenazas se vive el proceso de dejar de ser lo que se es y tener coraje para saltar a ser otros diferentes en la continuidad permanente del cambio (en otras palabras, hacer de la posibilidad una potencia de crear), ¿cómo deben operarse dichos saltos, de tal forma que al vivir las experiencias devengan en fuerzas comunitarias de resistencias?

Para responder la pregunta, es preciso comprender cómo el acto de exponerse a lo posible y sentir el riesgo de vivir en comunidad, lleva implícito el "cuidar". Espósito señala cómo el cuidado hace referencia no a cargar con el otro, ni asistirlo, a acompañarlo a ser coherente con la vida (Espósito, 2009). Ello remite al ejercicio de una ética del cuidado donde la sinceridad consigo mismo, antes que con un amo o una autoridad, es el fundamento de la acción. Luego, vivir en comunidad pone en juego el riesgo de ser sincero consigo mismo de cara a los demás.

Es entonces un "cuidar" con el otro, un circular por los acontecimientos - lo que sucede, lo que pasa, lo posible por pasar-; es ocuparse o cuidar de sí mismo, no para ir en búsqueda de esencialismos o sujetos racionales, o de lugares epistemológicos, sino para retornar al éthos. $\mathrm{Al}$ asumir la pregunta “¿cómo vivo?” se emprende el camino de mirar lo que sucede en un aquí y en un ahora, con la actitud de la no mentira, en la que la palabra acontece de manera sincera. Esto corresponde a aquellos actos donde la comunidad, pone a prueba la sinceridad de las propuestas de cara al otro o los otros, en los espacios singulares de la grupalidad y en las tareas propias de la acciones, con lo que cobra sentido la palabra en lo comunitario en tanto con ella se realiza uno de los actos de cuidar la forma y las maneras de construir saberes que no guarden pretensión de dominio ni de regularizar a otros saberes, sino que recobren el sentido de lo vital del habla, allí donde la forma y manera de hablar pongan en riesgo el sentido de la existencia.

En otras palabras, lo anterior es comprender la palabra acto desde el ejercicio cotidiano de la sinceridad, en el que la comunidad posibilite el salto ético de hacerse sinceridad consigo mismo para devenir con los otros en el acto de los acontecimientos de la experiencia y de las subjetividades. Es esto la actitud foucaultiana de la parresia: la manera de tomar el coraje de ser veraz, de decir y exponer el saber-verdad como el acontecer de las pasiones, como el dar la palabra con la fuerza de la oralidad que no busca ser verdad, sino ser afectivamente veraz, y que recobra la coherencia de la opinión.

La parrhesía es pues cierta manera de hablar [...] es una manera de decir la verdad [...] es una manera de decir la verdad de tal modo que, por el hecho mismo de decirla, abrimos, nos exponemos a un riesgo [...] es una manera de abrir ese riesgo ligado al decir veraz al constituirnos en cierta forma como interlocutores de nosotros mismos cuando hablamos, al ligarnos al enunciado y la enunciación de la verdad. Para terminar, la parrhesía es una manera de ligarnos a nosotros mismos en la forma de un acto valeroso. Es el libre coraje por el cual uno se liga a sí mismo en el acto de decir la verdad. E incluso es la ética del decir veraz, en su acto arriesgo y libre (Foucault, 2009, p. 82).

Esto es la parresia: poner en juego el ejercicio de ocuparse de sí mismo de cara a esos otros posi- 
bles de ser comunidad. Es, ante todo, una relación en la que se instaló el reto de lo veraz con la palabra que se ubica más allá de lo dialógico, del lenguaje, de la sintaxis de esos logos ordenadores de saberes normalizados por metodologías que dicen cómo hablar y cómo escribir, para los cuales la actitud ética de la palabra en comunidad pierde relevancia y se convierte en un artefacto al servicio del poder. Es restituir la fuerza de la palabra como acto ético de ser constructora de una política amiga de la palabra acto, que al circular en lo cotidiano hace de los procesos formativos el centro de preocupación de una pedagogía de la experiencia. Es allí donde la pedagogía es la cuidadora en la comunidad de la palabra acto, como acontecimiento ético de ser constructora de una política de la amistad. Se trata de una pedagogía cuidadora de la palabra que se hace diciéndose y exponiéndose a los otros, que no busca definiciones, sino que provoca la pluralidad de los encuentros de saberes dispuestos a darse lugares que no son fijos ni determinantes.

La palabra dicha como una palabra no fija sino fluida, una palabra que es "en sí y por sí" sino que deviene, una palabra que no parece en la forma "de lo que fue dicho" sino en la forma de lo que viene diciéndose, de lo que diciéndose viene, quizás de lo aún por decir, y una palabra por último que no es inanimada sino que está animada, viva (Larrosa, 2003, p. 44).

La anterior es una palabra viva, oída del acontecimiento, que no explora un destino, pero se compromete con él; que no predefine una llegada pero desea construir un sendero; que ofrece al otro lo posible y es fabricadora de autonomías como producto de los encuentros con los otros y con las subjetividades grupales.

Por eso, la palabra oída es la palabra "que no está destinada": no la palabra que nos construye como destinatarios, es decir, la que se propone hacer alguna cosa con nosotros, ni tampoco la que nosotros buscamos desde nuestras preguntas, o desde nuestras inquietudes, desde lo que queremos en definitiva, sino aquella en la que oímos "la voz del destino" (Larrosa, 2003, p. 44).
La palabra de oídas acontece en lo comunitario, atravesada por el amor en tanto pasión. No da la escritura sujeta a la lógica de los conceptos preestablecidos; en lugar de ello, da el sentir abierto del amor-pasión para dejar que el pensamiento se abra y se exponga a lo impredecible de los afectos. Estos permiten voces que otorgan el sentido de sus tonalidades, haciendo de la palabra no solo actos verbales sino preverbales, en lo rítmico de las tonalidades emotivas y volitivas de los acontecimientos. Es decir, la palabra acto en la comunidad es oralidad: es la palabra ruido. Está abierta a la fecundidad del dar a lo posible del encuentro con el otro, o los otros en donde no hay límites; donde entre más se vive el acto de dar y de cuidar, más se potencializa el querer dar sentido a la existencia; donde el saber es la opinión comprometida con el devenir de la palabra en lo grupal.

En tanto acto, la palabra acepta la lucha entre los saberes, pero no para someter, ni para la erudición de los doctos. Se trata de una palabra afectada por las experiencia vividas que se expone para diluir, en los procesos de sinceridad, aquel sujeto de palabra: es experiencia, y riesgo; es arrastrar el sujeto a dejar de ser y es sentir lo que sucede.

Desde el punto de vista de la experiencia, lo importante no es ni la posición (nuestra manera de ponernos), ni la o-posición (nuestra manera de oponernos), ni la imposición (nuestra manera de imponernos), ni la pro-posición (nuestra manera de proponernos), sino la ex -posición, nuestra manera de ex - ponernos, con todo lo que ello tiene de vulnerabilidad y de riesgo. Por eso es incapaz de experiencia el que pone, o se opone, o se impone, o se propone, pero no se ex -pone (Lazzarato, 2006, p. 175).

En otras palabras, es una pedagogía inquieta, que no se preocupa por decir al otro o los otros qué decir y hacer, sino por hacer de la palabra la aguja que actúa y se dirige a la formación; no a la información ni a los saberes epistémicos - como diría Freire, a los saberes doctos-, sino a la aventura del encuentro con los otros para ser multitud. 


\section{El conversatorio, escenario de la pa-} labra acto

El conversatorio, que etimológicamente significa vivir y dar vueltas en compañía, pero también evoca el convertirse otros siendo uno, es uno de los lugares en que se asumen las experiencias de vidas y se tiene el coraje de decir; es allí donde se expone la verdad, sucede el acto de la sinceridad comunitaria y los procesos pedagógicos tienen la posibilidad de acompañar lo formativo del hecho de cuidar la palabra acto.

Antes de proseguir, es necesario comprender la relación entre verdad y opinión, ya que esta última hace parte de la palabra. La verdad en el conversatorio no se da, ni se dice, ni se dictamina; acontece, de manera paradójica, a través de la opinión. No busca ser verdad sino que viene y sucede con veracidad. Ahora bien, lo que ha sucedido con la opinión en la historia del conocimiento, e inclusive en la política, es que se la ha cargado con la marca de la falsedad y el error, y por ende, ha sido relegada al plano de la distorsión de los sentidos de la vida cotidiana, en tanto que es sometida a saberes validados por los poderes dominantes de regularización metodológica y normalidad política. Lo que sucede en el conversatorio es la restitución de la pluralidad y de la coherencia de la opinión, que al mostrarse a los demás logra ser veraz, como lo afirma Hannah Arendt, recobra el sentido de la doxa, de ser pluralidad, de exponer lo que cada individuo siente, y tiene como verdad, no solo a sí mismo sino a la pluralidad.

La palabra doxa no significa meramente opinión, sino también esplendor y fama. Como tal, está en relación con el espacio político, que es la esfera pública, en la que cada cual puede aparecer y mostrar quién es. Declarar la propia opinión guardaba relación con ser capaz de mostrarse uno mismo, de ser visto y oído por los demás (Arendt, 1997, p. 52).

Por lo anterior, la palabra acto se asoma a lo exterior desde la opinión. No es la opinión banal ni esmerada por demostrar conocimientos sobre lo que más se sabe, sino aquella que está ligada con el hecho de ser veraz y ponerse de cara al otro. Es mirar al otro no como igual ni varios sino como posibles; la fuerza de la comunidad permite esto último, en tanto imprime el coraje de arrogarse a ser coherente con lo que se dice y se hace. Es aquella palabra cómplice con el sí mismo, que sabe sentir el compromiso de lo que está sucediendo al momento de enunciarse. Es aquella que al decirse está de frente con lo que dice, porque sabe que solo así la pedagogía es experiencia - esto es, poner en situación el acto de cuidar de la palabra acto-.

Así entonces, en el conversatorio suceden dos actos de la palabra. De un lado lo ético, en el cuidar la coherencia de la forma y la manera de decir la opinión venida del mundo cotidiano, y oída en los escenarios comunitarios, para ser transformada en pensamiento comprometido con la existencia. $\mathrm{Y}$ de otro, el tejer un discurso y unos saberes que en la conversación con los otros - de oídas- confronta la forma como se viven las experiencias; esto es, retornar hacia sí mismo pero no de manera aislada sino con todos, lo que requiere el rigor de estar atento a lo que se dice y se hace, sin que nadie actúe como evaluador o juez.

Dicho de otra manera, el conversatorio va más allá del ejercicio dialógico de dos sujetos — llámense educando o educador, o profesional o comunidad-, en tanto no se conversa con una persona definida que tiene identidad. Se conversa, ante todo, con el sentido de la existencia, lo que permite instantes de conversión donde esta última, en tanto vivencia, hace que en el conversatorio no se enseñe ni se instruya, sino que se acoja y provoque el venir de un pensamiento comprometido con las propuestas potencializadoras de la vida en comunidad. Es entonces cuando emerge una inquietud: ¿existe una estructura pedagógica en el conversatorio?

Quizá no se asiste a estructuras sino a empatías al disponerse a entrar en la conversa con otros, de oídas, en la oralidad tonal de las palabras coherentemente pasionales. Se instalan intensidades de las formas y maneras de decir y de hacer, con lo que se visibilizan tres líneas existenciales: la primera, la situación vital, o el acontecimiento que se vive 
o trascurre, el cual pone en el borde el sentido de vida; la segunda, la pregunta, que como tábano, no busca juzgar, definir o predefinir un contenido, sino poner en situación, confrontar lo que se vive y cómo se vive; y la tercera, el devenir vital, es el acontecimiento de las transformaciones singulares de las condiciones humanas de vida y la acción misma de las palabras 'grupo' y 'comunidad', por ser libertaria.

Estas tres líneas no buscan ser estructurales ni estructurantes, ya que el contexto comunitario es devenir en las aventuras de las experiencias de las palabras actos. Es la fecundidad de dar vida con las palabras actos que, tejidas desde el oído y en las relaciones pasionales de ser amigo de las palabras, ofrecen la desnudez de la vida que ignora, en tanto se suceden de manera espontánea y no en la línea secuencial del hecho de contar los hechos que pasan.

La fecundidad es dar una vida que no será nuestra vida ni la continuación de nuestra vida porque será una vida otra, la vida del otro. O dar un tiempo que no será nuestro tiempo ni la continuación de nuestro tiempo porque será un tiempo otro, el tiempo del otro. O dar nuestra palabra que no será nuestra palabra ni la continuación de nuestra palabra porque será una palabra otra, la palabra del otro (Larrosa, 2003, pp. 36-37)

Así, comprender la primera línea como situación vital, es llevarla a la reflexión de las condiciones humanas de vida - que Arendt definió como labor, trabajo y acción-, pero no para ser interpretadas desde la necesidad y la carencia, sino desde el posarse en la acción, donde la palabra se sucede en la aventura de ser el poder constructor de un política comprometida con la pluralidad. En el ámbito del conversatorio, ello implica dos cuestiones: 1) restituir la experiencia no desde lo catastrófico sino desde la aventura, y disponerse a escucharla en el silencio como cómplice de los demás, esto es, mirar lo común como un posible; y 2) disponerse atentamente al encuentro con lo otro, con el otro. Esta situación es la que permite asomarse al exterior de la comunidad y sentir que está en las voces y en las tonalidades de las palabras que se expresan.
La segunda línea, la pregunta, es el aguijón que construye en el encuentro plural con los otros: no busca definir, imponer, solucionar, planificar, o dirigir un problema comunitario, sino que es la ocasión de sentir el poder del encuentro con los otros, en el cual desaparece el sujeto, el yo, la identidad. Corresponde a la pregunta que busca aclarar con la palabra y la pasión de la tonalidad de lo que se dice, tener la ocasión de llamar la atención sobre la forma de vivir, desde una ética de la palabra sincera y comprometida en hacer de la libertad actos de posibilidad. Por ello, la pregunta no se prepara, ni se obliga, ni se pretende; en lugar de ello, se llega a la oportunidad de los encuentros para recobrar el sentido que tiene la existencia. La pregunta es el acto mismo del dar, de ofrecerse para trasgredir lo que se es y subvertir lo instituido como único posible; es la palabra pacientemente sincera de lo singular, donde toma fuerza la construcción de escenarios comunitarios comprometidos con la palabra y con la vida.

En la tercera línea, el devenir vital - donde la palabra es acción propia-, suceden las mutaciones que colocan como reflexión crítica y activa la libertad, pero no jurídica sino posible. En palabras de Larrosa (2000) "la libertad es la experiencia de la no verdad, de la transgresión, del ir más allá de lo que somos, de la invención de nuevas posibilidades de vida" (p. 250). En otros términos, en las prácticas de los encuentros experienciales ya no existen sujetos sino multiplicidad de relaciones afectivas, las cuales que son subjetivaciones que vienen sin imponerse, cuyo sentido es la forma de vivir la vida desde el goce de la misma.

Una singularización existencial que coincida con un deseo, con un determinado gusto por vivir, con una voluntad de construir el mundo en el cual nos encontramos, con la instauración de dispositivos para cambiar los tipos de sociedad, los tipos de valores que no son nuestros (Guattari \& Rolnik, 2006, p. 29)

En esta línea vital se crea la democracia como un estilo de vivir, donde la palabra acto la saca de los ámbitos estaduales de la ley y la pone en el devenir de las acciones de la conversación. 
Sólo en una sociedad en la cual rija el poder de la palabra es posible la democracia [...] La democracia es palabra, logos, discurso [...] se construye la democracia conversando en un diálogo sin fin. La democracia es el intento de alejar la fuerza de poner, la razón y la no-razón como condición de convivencia [...] consiste en sacar la democracia del Estado y situarla en el mercado, en la escuela, en la calle, en la fábrica, en el ágora (Botero, 2001b, p. 709).

Con lo anterior, el hecho de recobrar la relación de la conversación con la palabra acto, en el ámbito de la comunidad, posibilita el ejercicio de una libertad transgresora y constructora de una democracia como estilo de vida. Esta acontece en la singularidad de los encuentros de la calle, del barrio, de las esquinas, del parque y de la vida escolar para tejer escenarios de convivencia - del acoger para vitalizar - donde lo mínimo sea ocuparse del ethos de exponer la opinión, a riesgo de que esta corresponda a saberes no regularizadores sino dispuestos a darse en la conversa sin fin por los sentidos de vivir.

Las tres líneas discutidas se dan en relaciones donde lo posible es permanente, para recordar que lo establecido no es absoluto y que la condición es creada, y donde la palabra deviene en la conversación. No se llega a esta última por obligación, imposición o necesidad, sino por el coraje de ponerse en frente del otro para hacer de la conversación un ejercicio espiritual, en el que esta haga del diálogo el ejercicio de estar en frente del otro y recobrar su sentido espiritual.

El diálogo sólo llega a ser verdaderamente en presencia ante otro y ante uno mismo. Desde este punto de vista todo ejercicio espiritual es diálogo en la medida en que supone una auténtico ejercicio de presencia, tanto ante uno mismo como ante otro (Hadot, 2000, p. 36).

Por lo anterior, con el conversatorio en la comunidad se asiste pedagógicamente a que el pensamiento venga de oídas y, en el ejercicio de darse en la experiencia de la palabra acto, se restituya el lugar "no lugar" de lo pedagógico: la formación vital de la vida, en lugar de la ciencia como juego discursivo de los saberes epistemológicos.
Este es el lugar de la pedagogía: ser la cuidadora de la palabra acto para desatar actitudes éticas y de convivencias comunitarias comprometidas con la potencia de vivir, de lo humano y lo no humano que hace la diferencia, a la vez que le corresponde ser la cuidadora de procesos de formación comunitaria. Empero, quizás a lo que se asiste en la comunidad como posible es al surgimiento de la figura de la amistad como aquel saber ser todo encubierto, que con la pregunta encuentra la oportunidad de formar amigos del pensamiento vivo, en el que la palabra deviene políticamente en comunidades autónomas al comprometerse con la vida; en estas, el centro no son los discursos ni las preocupaciones estatales, sino el coraje de atreverse a pensar con los otros lo posible, que corresponde a la creación.

\section{Conclusiones}

El recorrido realizado permite dejar una serie de trazos conceptuales que, lejos de estar concluidos, invitan a seguir reflexionándose y a ser posibles.

- La comunidad es el encuentro de experiencias donde la palabra acto, al acontecer en el conversatorio, tiene dos facetas: de un lado, es un ejercicio de ponerse frente al otro y, guardando coherencia entre el decir y el hacer, construir posibles trasgresores del presente instituido. Y de otro está la experiencia, esto es, el acontecimiento de lo que sucede, que hace de la comunidad una posibilidad, y que con el coraje de atreverse a ser sincero - en la conversa de oídas y con la pregunta como tábano-, permite que el pensamiento suceda en la permanente atención de la experiencia con el otro, para devenir en pluralidad. Es aquí donde la pedagogía se hace cuidadora de la palabra acto, en sus actitudes éticas de la sinceridad de la palabra frente a lo que acontece y de cara con la vitalidad de la vida. En momentos sociales y comunitarios donde pareciera que todo está dicho sobre la comunidad, o no hay nada que decir sobre ella, es necesario repensarlas más allá de lo estadual y de la racionalidad epistemológica, y retornar a 
reflexionar sobre sus procesos desde las singularidades existenciales encontradas en la pasión del gusto de vivir, con una voluntad de construir mundos posibles de pasiones alegres.

- Acompañar pedagógicamente los procesos comunitarios es tener claro que no se acompaña a la comunidad a decir qué hacer o proponer. En lugar de ello, se debe recobrar de lo pedagógico la fuerza de ser pensamiento vivo, en el que mediante el conversar y bajo la palabra acto, se ofrezca con la pregunta aquel escenario de acoger a esos otros para restituir el ser amigos de la vida y del pensamiento, antes que sabios o virtuosos. Allí radica lo vital de la comunidad: el hacer sentir mundos posibles desde lo simple que implica vivir la vida, en contraste con la profundidad que reviste darle sentido ético y de la política.

\section{Referencias}

- La palabra acto en la comunidad visibiliza la democracia como estilo y forma de vivir desde lo singular de los mundos cotidianos en los que, en tanto poder, la palabra posibilita construir formas de vivir juntos donde no se instalan únicos saberes y totalitarismos. Por ello, la pedagogía articula la ética y la política desde la amistad.

Así entonces, la democracia como forma de vivir implica restituir el lugar de la palabra ser poder de decidir en los destinos comunitarios, siendo los saberes sus devenires en la edificación de darse las formas de gobernar autónomamente. Queda el reto teórico de afectar a otros mundos posibles que, con propuestas comunitarias, deseen hacer de la palabra acto una experiencia de construcción de comunidades para la vida.
Arendt, H. (1993). La condición bumana. Barcelona: Paidós.

Arendt, H. (1997). La promesa de la política. Barcelona: Paidós.

Bajtin, M. (1997). Hacia un filosofía del acto ético. Barcelona: Anthropos.

Botero, D. (1999). Teoría social del derecho. Bogotá: Universidad Nacional de Colombia.

Botero, D. (2000). El derecho a la utopia. Bogotá: Universidad Nacional de Colombia.

Botero, D. (2001a). Vida, ética y democracia. Bogotá: Universidad Nacional de Colombia.

Botero, D. (2001b). El poder de la filosofía y la filosofía del poder. Bogotá: Universidad Nacional de Colombia.
Deleuze, G., \& Guattari, F. (1994). Mil Mesetas. Valencia: Pre-Textos.

Equipo Comunitario de Ladera para la Promoción de Vida y Salud (2012-2014). Conversatorios. Cali.

Espósito, R. (2003). Communitas. Origen y destino de la comunidad. Buenos Aires: Amorrortu.

Espósito, R. (2009). Comunidad, inmunidad y biopolitica. España: Herder.

Foucault, M. (2002). La hermenéutica del sujeto. México: Fondo de Cultura Económica.

Foucault, M. (2009). El gobierno de sí y de los otros. México: Fondo de Cultura Económica.

Foucault, M. (2010). El coraje de la verdad. México: Fondo de Cultura Económica. 
Gil, M. F. (2012). El cuidado, de cara a la ética, la política y la pedagogía vivencial para la vida. Revista Entramado, 8(1), 178-187.

Guattari, F., \& Rolnik, S. (2006). Micropolitica. Cartografia del deseo. Madrid: Traficantes de Sueños.

Hadot, A. (2006) Ejercicios espirituales y filosofía antigua. Madrid: Siruela.

Hardt, M., Negri, A. (2004). Multitud. Barcelona: Debates.
Larrosa, J. (2000). Pedagogía profana. Buenos Aires: Novedades Educativas.

Larrosa, J. (2003). Entre lenguas. Barcelona: Laertes.

Lazzarato, M. (2006). Por una politica menor. España: Traficantes de Sueños.

Lazzarato, M. (2007). La filosofía de la diferencia. Bogotá: Universidad Central. 